\title{
En búsqueda de una mejor definición y estrategia diagnóstica para la lesión renal aguda: una nueva propuesta
}

\author{
Looking for a better definition and diagnostic strategy for acute kidney injury: a new \\ proposal
}

Si bien existen diversas definiciones de lesión renal aguda (LRA), clásicamente, se la define como un grupo heterogéneo de afecciones caracterizadas por una disminución repentina en la tasa de filtración glomerular (TFG), expresada como un aumento en la creatininemia (creatininemia $\geq 0,3 \mathrm{mg} / \mathrm{dl}$ en 48 horas o creatininemia $\geq 1,5$ veces respecto de su valor basal dentro de los primeros 7 días) o una reducción del volumen de orina (diuresis $<0,5 \mathrm{ml} / \mathrm{kg} / \mathrm{h}$ por 6 horas). ${ }^{1,2}$ Sin embargo, esta definición no refleja con exactitud la fisiopatología del síndrome, ya que una LRA puede limitarse a los túbulos sin comprometer los glomérulos y, por lo tanto, no reducir la TFG del paciente ni su diuresis. Este fenómeno se observa clínicamente en algunas lesiones renales inducidas por fármacos (ej.: aminoglucósidos) o en casos de isquemia renal parcial, puesto que la médula renal es más sensible a la hipoxia que la corteza renal. ${ }^{3,4}$ Por lo tanto, la definición actual de LRA puede entorpecer su diagnóstico en situaciones clínicas particulares.

Hay dos inconvenientes adicionales derivados de la definición actual de LRA. En primer lugar, el diagnóstico de LRA no oligúrica se basa en la detección de la reducción de la TFG; sin embargo, el marcador diagnóstico habitualmente utilizado para tal fin es la creatininemia, la cual es un marcador tardío, debido al hecho de que aumenta sus niveles séricos recién cuando se produce una reducción de la TFG de, al menos, el $50 \%$. Este hecho conduce de manera inexorable a un diagnóstico tardío de LRA y, por ende, a un tratamiento tardío y peor pronóstico., ${ }^{1,3}$

En segundo lugar, a pesar de haberse descrito nuevos biomarcadores de LRA, su disponibilidad es reducida, tienen especificidad baja, costo elevado, utilidad no universal y, principalmente, no garantizan la detección inmediata de la LRA, ya que su solicitud depende de la sospecha clínica de esta entidad por parte del médico, que está supeditada a la definición de LRA actualmente utilizada. ${ }^{5,6}$

A fin de superar estas dificultades, se presentan aquí las siguientes propuestas, válidas tanto para el paciente adulto como el pediátrico.
Primero, cambiar la definición actual del diagnóstico de LRA por una más completa: La LRA es un grupo heterogéneo de afecciones que se caracteriza por una alteración repentina de la función tubular, disminución de la TGF o reducción del volumen de orina. La idea de modificar la definición no sería por una mera razón académica, sino, fundamentalmente, pragmática, ya que llevaría al médico a evaluar la función glomerular y también la tubular para descartar la presencia de la afección, y esta evaluación más meticulosa contribuiría a una detección más temprana de este síndrome.

Segundo, basar el diagnóstico temprano de LRA no solo en el monitoreo de un marcador sérico de TFG, sino también en un marcador funcional tubular urinario, por ejemplo, la excreción fraccional de sodio (EFNa), y se requiere, además, realizarlo antes de la instalación de la LRA: monitoreo renal preventivo. Por supuesto, se propone que esta estrategia se aplique a pacientes con alto riesgo de LRA (por ejemplo: pacientes con cuidados críticos o personas con fármacos o afecciones potencialmente lesivas para el parénquima renal). La idea sería no buscar un valor absoluto particular del índice urinario, como es el caso de los clásicos índices urinarios de Miller, o distinguir entre LRA prerrenal y renal, sino detectar un cambio significativo (aumento o disminución) en un valor de índice urinario respecto de sus valores basales. Se podría argumentar que estos cambios en los índices urinarios evaluados de manera preventiva podrían ser causados por meras fluctuaciones hemodinámicas; sin embargo, un adecuado test de rastreo debe ser sensible y, por ende, puede presentar positivos falsos. Además, estas fluctuaciones hemodinámicas, si no se detectan temprano y se manejan de modo adecuado, pueden llegar a inducir daño isquémico renal. Por lo tanto, la detección temprana de estos estados clínicos riesgosos mediante la aplicación de la monitorización renal preventiva podría ayudar, precisamente, a evitar la LRA. En este sentido, existen datos preliminares que pueden respaldar esta idea, los cuales proceden de un estudio prospectivo que se está realizando en 
la actualidad. En este protocolo, se obtuvo una EFNa diaria en todos los pacientes ingresados en la Unidad de Cuidados Intensivos desde su ingreso, y aquellos que presentaron un cambio significativo en la EFNa el día anterior (día -1) del diagnóstico clínico de LRA (día 0) desarrollaron, finalmente, LRA. La monitorización renal preventiva podría detectar de manera temprana la aparición de la LRA, justo en un momento en que una evaluación exhaustiva de su estado de volumen y la medicación potencialmente nefrotóxica podrían evitar o mitigar este síndrome. ${ }^{7}$ Desde ya, son bienvenidos los investigadores de otros centros que quisiesen sumarse a nuestro estudio.

En conclusión, se propone modificar la definición de la LRA, así como su estrategia diagnóstica, incluidos los conceptos de disfunción tubular aguda y vigilancia renal preventiva, respectivamente, a fin de poder realizar un diagnóstico más amplio y temprano de este síndrome.

Dr. Carlos G. Musso ${ }^{a, b}$, Mg. Sergio Terrasa ${ }^{c}$, Mariana Ciocchini ${ }^{d}$ Mg. Henry González-Torres ${ }^{e}$ y Dr. Gustavo Aroca-Martínez

a. Departamento de Fisiología, Instituto Universitario del Hospital Italiano de Buenos Aires, Argentina.

b. Unidad de Biología del Envejecimiento, Hospital Italiano de Buenos Aires, Argentina.

c. Instituto Daomi afiliado a la Facultad de Medicina, Universidad de Buenos Aires, Buenos Aires, Argentina. d. Servicio de Medicina Familiar, Hospital Italiano de Buenos Aires, Argentina.

e. Clínica de la Costa, Universidad Simón Bolívar, Barranquilla, Colombia.

Correspondencia: carlos.musso@hospitalitaliano.org.ar

http: / / dx.doi.org/10.5546/ aap.2019.4

Texto completo en inglés:

http: / / dx.doi.org/10.5546/ aap.2019.eng.4

Cómo citar: Musso CG, Terrasa S, Ciocchini M, González-Torres H, et al. En búsqueda de una mejor definición y estrategia diagnóstica para la lesión renal aguda: una nueva propuesta. Arch Argent Pediatr 2019;117(1):4-5.

\section{REFERENCIAS}

1. Levey AS, James MT. Acute Kidney Injury. Ann Intern Med. 2018; 168(11):837.

2. KDIGO Clinical Practice Guideline for Acute Kidney Injury. Kidney Int. 2012; 2(1):1-138.

3. Rennke H, Denker B. Renal Pathophysiology. Philadelphia: Lippincott Williams \& Wilkins; 1994.Págs.267-90.

4. Musso CG. Aminoglycosides and nephropathy. Rev Electron Biomed. 2004; 2:2-4.

5. Beker BM, Corleto MG, Fieiras C, Musso CG. Novel acute kidney injury biomarkers: their characteristics, utility and concerns. Int Urol Nephrol. 2018; 50(4):705-13.

6. Pacheco-Lugo L, Díaz-Olmos Y, Aroca-Martínez G. Biomarkers in biological fluids and their potential use as indicators of lupus nephritis in individuals with systemic lupus erythematous. Rev Colomb Nefrol. 2016; 3(1):39-47.

7. Musso CG, Silva D, Arroyo M, Propato F, et al. Renal monitoring in intensive care unit. Abstract presentado en XII Congreso Internacional de la ISHD; del 18 al 21 de abril de 2018. Cartagena de Indias, Colombia. 2018.

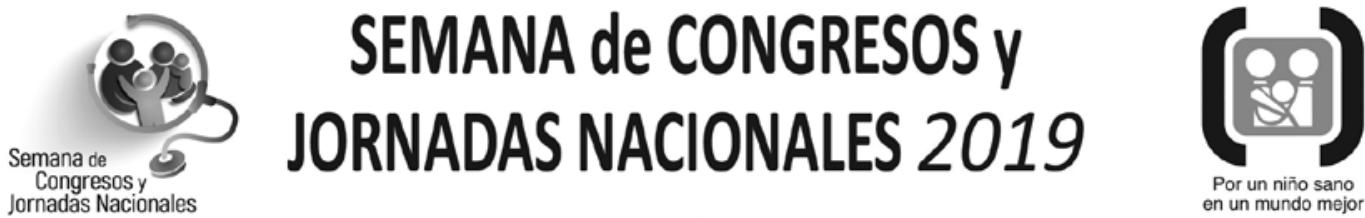 \\ - $4^{\circ}$ Congreso Argentino de Neonatología \\ - $2^{\circ}$ Congreso Argentino de Medicina Interna Pediátrica \\ - $10^{\circ}$ Congreso Argentino de Salud Integral del Adolescente \\ - $1^{\circ}$ Congreso Argentino de Alergia e Inmunología en Pediatría \\ - $5^{\circ}$ Jornadas Nacionales de Actividad Física y Deportiva en el Niño y el Adolescente \\ - $4^{\circ}$ Jornadas Nacionales de Auxología - $1^{\circ}$ Jornadas Nacionales de Desarrollo \\ - $1^{\circ}$ Jornada Nacional de Ética y Humanidades}

20, 21, 22, 23 y 24 de mayo de 2019

Sedes: Panamericano Buenos Aires Hotel \& Resort • Carlos Pellegrini 551

EXE Hotel Colón • Carlos Pellegrini 507

Ciudad Autónoma de Buenos Aires 